\title{
Hepatitis aguda por virus E con características de autoinmunidad. Reporte de un caso
}

\author{
María Laura Garrido, ${ }^{1}$ María Laura Reyes Toso, ${ }^{2}$ Sebastián Raffa, ${ }^{3}$ Valeria Inés Descalzi ${ }^{2}$ \\ ${ }^{1}$ Hospital San Luis. Provincia de San Luis, Argentina. \\ ${ }^{2}$ Hospital Universitario Fundación Favaloro. Ciudad Autónoma de Buenos Aires, Argentina. \\ ${ }^{3}$ Hospital Privado de Comunidad. Mar del Plata, Argentina.
}

Acta Gastroenterol Latinoam 2021;51(2):217-222

Recibido: 05/06/2020 / Aceptado: 10/05/2021 / Publicado online: 21/06/2021 / https://doi.org/10.52787/dtzy3014

\section{Resumen}

Analizamos el caso de un paciente masculino de 49 años que presentó signos clinicos de hepatitis aguda con una sospecha inicial de etiología autoinmune. Los hallazgos del laboratorio demostraron un anticuerpo antinuclear positivo, un anticuerpo antimúsculo liso y una gammaglobulina sérica alta. La histología de la biopsia hepática reveló cambios compatibles con una hepatitis autoinmune que, asociada con un puntaje del Grupo Internacional de Hepatitis Autoinmune de 7, determinó el inicio del tratamiento con Meprednisona y Azatioprina. Durante el seguimiento, recibimos resultados serológicos positivos de inmunoglobulina $M$ contra el virus de la hepatitis E con viremia detectable, mediante la técnica de reacción en cadena de la polimerasa de transcripción in-

Correspondencia: María Laura Garrido Héroes de Malvinas $N^{\circ} 225$. San Luis, Argentina Tel.: 0341152011049

Correo electrónico: mlgarrido1984@gmail.com versa, cambiando el diagnóstico a hepatitis aguda secundaria al virus E. Se suspendió la inmunosupresión y el paciente continuó con mejoría clínica y bioquímica. En Argentina, las pruebas para la detección de virus de la hepatitis E no se realizan de forma rutinaria. Sin embargo, para evitar un diagnóstico erróneo, esta etiología debe descartarse en pacientes con hepatitis aguda antes de etiquetarla como autoinmune. Esto reduciria un tratamiento innecesario que podría poner en peligro a los pacientes.

Palabras claves. Hepatitis autoinmune, virus de la hepatitis E, hepatitis aguda.

\section{Acute Hepatitis "E" Virus with Features of Autoimmunity. A Case Report}

\section{Summary}

We analyze the case of a 49-year-old male patient who presented with clinical signs of acute hepatitis with an initial suspicion of autoimmune etiology. Laboratory findings demonstrated positive antinuclear antibody, anti-smooth muscle antibody and high serum gamma globulin. Histology of the liver biopsy revealed changes compatible with autoimmune hepatitis, which associated with an International Autoimmune Hepatitis Group score of 7 , determined the initiation of treatment with Meprednisolone and Azathioprine. During the follow-up, we received positive serological 
results of immunoglobulin $M$ against hepatitis $E$ virus with detectable viremia by reverse transcription polymerase chain reaction technique, changing the diagnosis to acute hepatitis secondary to hepatitis E virus. Immunosuppression was suspended and the patient continued with clinical and biochemical improvement. In Argentina, hepatitis E virus testing is not routinely performed, however, to avoid misdiagnosis, this etiology should be ruled out in patients with acute hepatitis before labeling it as autoimmune hepatitis. This could reduce unnecessary treatment that could endanger our patients.

Keywords. Autoimmune hepatitis, Hepatitis E Virus, Acute hepatitis.

\section{Abreviaturas}

ANA: Anticuerpo antinuclear.

ASMA: Anticuerpo antimúsculo liso.

ANTI-LKM: Anticuerpos microsomales de hígado y riñon. ARN: Acido Ribonucleico

HAI: Hepatitis autoinmune.

IAHG: Grupo Internacional de Hepatitis Autoinmune.

IgG: Inmunoglobulina $G$.

VHE: Virus de la hepatitis E.

RT-PCR: Reacción en cadena de la polimerasa de transcripción inversa.

AST: Aspartato aminotransferasa.

ALT: Alanina aminotransferasa.

Ull: Unidades por litro.

GGT: Gamma glutamil transpeptidasa.

FAL: Fosfatasa alcalina.

RIN: Razón internacional normatizada.

VHB: Virus de la Hepatitis $B$.

VHA: Virus de la Hepatitis A.

VHC: Virus de la Hepatitis $C$.

VEB: Virus Epstein-Barr.

CMV: Citomegalovirus.

VIH: Virus de inmunodeficiencia humana.

TSH: Tirotrofina.

\section{Introducción}

El cuadro de hepatitis aguda puede ser secundario a múltiples causas, dentro de las cuales las infecciones virales, las hepatitis autoinmunes y enfermedades tóxicas o metabólicas son las más frecuentes. Una anamnesis exhaustiva en cada caso, incluido el aspecto epidemiológico, es una herramienta fundamental para la adecuada orientación etiológica.

En Argentina, el diagnóstico de hepatitis secundaria al virus $\mathrm{E}$ (VHE) era históricamente infrecuente. Sin em- bargo, desde hace unos años, el número de casos diagnosticados por esta entidad se encuentra en aumento.

El VHE es un virus ARN de la familia Hepeviridae que posee 4 genotipos con reconocidas diferencias en sus características clínicas y epidemiológicas. ${ }^{1}$ La presentación clínica de la infección por este virus es variable, y puede presentar desde cuadros subclínicos hasta una falla hepática fulminante.

Aún hoy, el estudio serológico y virológico para el VHE no se realiza de forma rutinaria en nuestro medio, aunque es necesario descartar esta infección sobre todo en casos de hepatitis aguda de origen incierto.

Se presenta el caso de un paciente con hepatitis aguda secundaria al VHE con un diagnóstico inicial autoinmune (HAI).

\section{Caso clínico}

Un paciente masculino de 49 años ingresó, en nuestro hospital, con una astenia progresiva y anorexia, asociadas a una alteración del hepatograma, de 3 semanas de evolución. El cuadro comenzó con dos episodios de fiebre que no se repitieron. Tenía antecedentes médicos de esclerosis múltiple, hipertensión arterial e infecciones repetidas del tracto urinario. No tenía antecedentes de ingesta etílica y su medicación crónica estaba compuesta por: nitrofurantoina $100 \mathrm{mg} /$ día, baclofeno $25 \mathrm{mg} /$ día, aminopiridina $10 \mathrm{mg} /$ día, citalopram $20 \mathrm{mg} /$ día, levetiracetam $1000 \mathrm{mg} /$ día, quetiapina $25 \mathrm{mg} /$ día, interferón beta- $1 \mathrm{~b}$ y ranitidina $150 \mathrm{mg} /$ día. Todos los medicamentos se suspendieron 21 días antes del ingreso, debido a anomalías en el laboratorio. En el examen físico, no se encontraron signos clínicos de encefalopatía ni de una enfermedad hepática crónica. Las pruebas de laboratorio mostraron: aspartato aminotransferasa (AST) $1935 \mathrm{U} / \mathrm{l}$ (10-50), alanina aminotransferasa (ALT) $1265 \mathrm{U} / \mathrm{l}$ (5-40), bilirrubina total 1,6 mg/dL (0,2-1); bilirrubina directa $1(0,1-0,5)$, gamma glutamil transpeptidasa (GGT) $708 \mathrm{U} / 1$ (7-51), fosfatasa alcalina (FAL) $174 \mathrm{U} / \mathrm{l}$ (40-150), tiempo de protrombina (quick) 74\% (70-120\%), RIN 1,3; factor V: $125 \%$ (70-120), factor VII: 70\% (70-120), inmunoglobulina G (IgG) $2200 \mathrm{mg} / \mathrm{dl}$ (549-1584), proteinograma por electroforesis con hipergammaglobulinemia policlonal (gammaglobulinas 2,3 g/dL); ANA positivo 1/320 (patrón nuclear homogéneo); ASMA positivo 1/80; AMA y anticuerpo anti-LKM negativo. La serología viral fue negativa para: Hepatitis B (VHB) (HBsAg, $\mathrm{HBeAg}$, anti-HBc, anti-HBs), Hepatitis A (VHA) (IgM e IgG), Hepatitis C (VHC) (Anti VHC y ARN por PCR-RT), virus Epstein-Barr (VEB) (IgM e IgG) y citomegalovirus (CMV) (IgM e IgG) (Tabla 1). También, se solicitaron 
otras determinaciones: niveles de hierro, ceruloplasmina, alfa-1 antitripsina, TSH, T3 y T4, que mostraron resultados normales; los anticuerpos antiperoxidasa, la antitiroglobulina y los anticuerpos contra la enfermedad celíaca fueron negativos. La serología para VHE ( $\operatorname{IgM}, \operatorname{IgG}$ ) fue diferida y no estaba disponible al momento de la admisión.

La ecografía doppler abdominal fue normal. Se le realizó una biopsia hepática percutánea, que mostró un infiltrado inflamatorio linfoplasmocitario con un compromiso periportal extenso. En el lobulillo, se observaron varios focos de balonización y un infiltrado moderado inflamatorio plasmocitario y linfocitario, con cuerpos de Councilman y formación de pseudorosetas. Con tricrómico, se identificó una fibrosis sinusoidal y periportal leve. La puntuación METAVIR fue A2-F1 (Figuras 1 y 2). Estos hallazgos fueron compatibles con una HAI. El puntaje simplificado del IAIHG fue de 7 puntos: ANA y ASMA, ambos positivos $>1 / 80(+2), \mathrm{IgG}>$ límite superior normal $(+1)$, biopsia hepática típica $(+2)$ y ausencia de hepatitis viral (+2). Debido a que el paciente cumplía con los criterios histológicos y el IAHG de 7 , se decidió comenzar un tratamiento con meprednisona de $60 \mathrm{mg} /$ día. El paciente evolucionó con el descenso progresivo de transaminasas y niveles de IgG. Se inició la reducción gradual de la dosis esteroidea y, a los 15, días se agregó azatioprina $50 \mathrm{mg} /$ día, con la normalización de los parámetros del laboratorio (Tabla 1). Los resultados de las pruebas serológicas para el VHE se obtuvieron después de que el paciente fuera dado de alta, mostrando una serología de IgM posi-

Figura 1. Biopsia hepática. Coloración: Hematoxilina-eosina. En el lobulillo se observan varios focos de balonización (flecha azul) y moderado infiltrado inflamatorio plasmocitario y linfocitario (flecha negra).

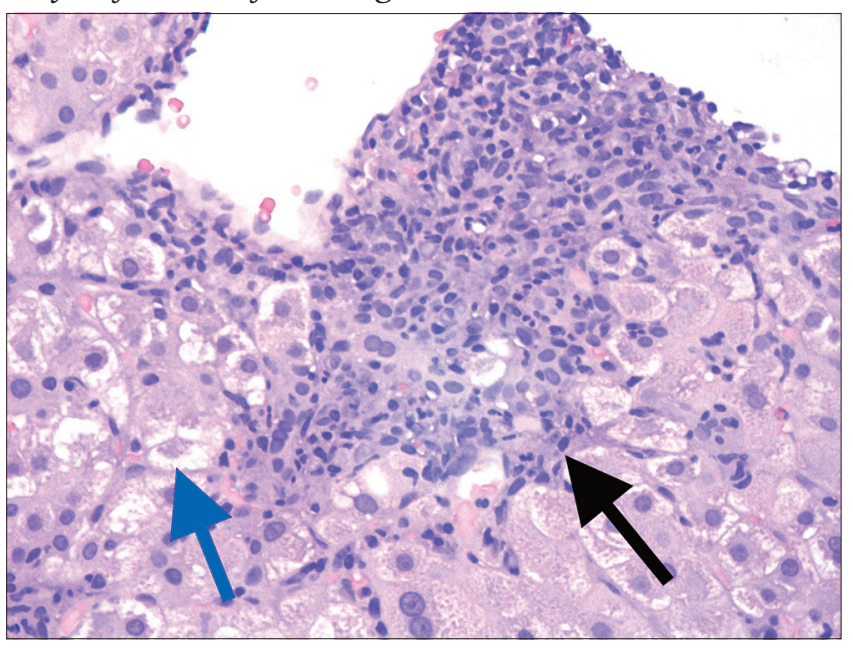

Figura 2. Biopsia hepática. Coloración: Hematoxilinaeosina. Infiltrado inflamatorio linfoplasmocitario lobulillar (flecha negra). Formación de pseudorosetas (flecha azul).

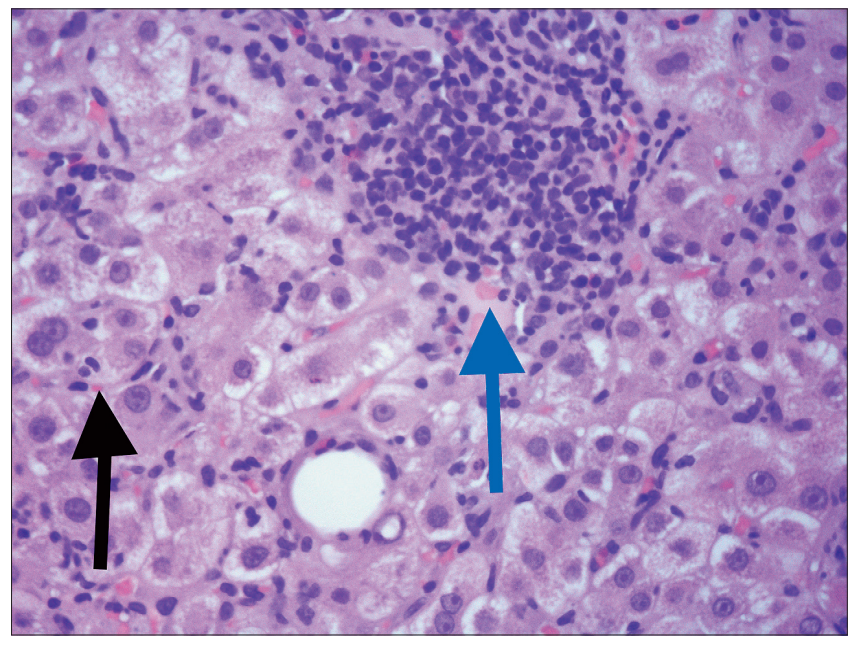

Tabla 1.

\begin{tabular}{|c|c|c|c|}
\hline Admisión & 3 semanas & 12 semanas & 6 meses \\
\hline ALT 1935 U/I & ALT $47 \mathrm{U} / \mathrm{I}$ & ALT $40 \mathrm{U} / \mathrm{I}$ & ALT $42 \mathrm{U} / \mathrm{I}$ \\
\hline AST $1265 \mathrm{U} / \mathrm{I}$ & AST $25 \mathrm{U} / \mathrm{l}$ & AST $34 \mathrm{U} / \mathrm{I}$ & AST $30 \mathrm{U} / \mathrm{I}$ \\
\hline $\begin{array}{l}\text { Bil T: } 1,6 \text { mg/dl } \\
\text { (directa: } 1 \mathrm{mg} / \mathrm{dl} \text { ) }\end{array}$ & Bil T: 1 mg/dl & Bil T: 1 mg/dl & Bil T: 0,8 mg/dl \\
\hline GGT: $708 \mathrm{U} / \mathrm{I}$ & GGT: $60 \mathrm{U} / \mathrm{l}$ & & GGT: $39 \mathrm{U} / \mathrm{l}$ \\
\hline FAL 174 U/I & FAL $120 \mathrm{U} / \mathrm{I}$ & FAL $100 \mathrm{U} / \mathrm{I}$ & FAL $120 \mathrm{U} / \mathrm{l}$ \\
\hline TP 74\% & TP 84\% & & TP 80\% \\
\hline $\operatorname{lgG} 2200 \mathrm{mg} / \mathrm{dl}$ & $\operatorname{lgG} 1200 \mathrm{mg} / \mathrm{dl}$ & & $\lg G 780 \mathrm{mg} / \mathrm{dl}$ \\
\hline $\begin{array}{l}\text { Gamaglobulinas } \\
2,3 \mathrm{~g} / \mathrm{dl}\end{array}$ & $\begin{array}{l}\text { Gamaglobulinas } \\
1,3 \mathrm{~g} / \mathrm{dl}\end{array}$ & & \\
\hline ANA $(+) 1 / 320$ & & ANA (+) 1/160 & ANA (-) \\
\hline ASMA 1/80 & & ASMA (-) & ASMA (-) \\
\hline \multicolumn{4}{|l|}{ VHB, VHA, VHC (-) } \\
\hline \multirow[t]{3}{*}{ VHE: pendiente } & IgG VHE (-), & $\lg \mathrm{V}$ VHE (+), & \\
\hline & $\operatorname{lgM}$ VHE (+) & IgM VHE (-) & \\
\hline & $\begin{array}{l}\text { PCR VHE } \\
\text { sangre/materia } \\
\text { fecal }(+)\end{array}$ & $\begin{array}{l}\text { PCR VHE } \\
\text { sangre (-) }\end{array}$ & $\begin{array}{l}\text { PCR VHE } \\
\text { sangre (-) }\end{array}$ \\
\hline
\end{tabular}

tiva para el VHE y la IgG negativa. Sobre la base de estos resultados, se le solicitó la RT-PCR en sangre y heces, siendo el VHE positivo en ambas. En este contexto, el caso se reinterpretó como una hepatitis aguda secundaria al VHE, por lo que se decidió suspender de forma 
progresiva la terapia inmunosupresora hasta su retirada completa y continuar con controles del hepatograma, la RT-PCR y la serología viral para el VHE.

Doce semanas después del inicio del cuadro, se repitió la serología viral del VHE: IgG VHE positiva, IgM VHE negativa, VHE en la RT-PCR negativo en sangre y heces, el ANA permaneció positivo con el mismo título y el ASMA negativo e IgG dentro de los límites normales. El paciente continuó con hepatograma normal durante la monitorización y 6 meses después de suspender la medicación, presentó enzimas hepáticas normales, IgG normal y los dos autoanticuerpos negativos. Luego de un año de seguimiento, se le realizó una nueva biopsia para descartar un diagnóstico incierto de hepatitis autoinmune. La histología del hígado fue normal y no se encontraron características patológicas (Figura 3).

Figura 3. Biopsia hepática. Coloración: Hematoxilina-eosina. Espacio porta sin hallazgos patológicos (flecha negra).

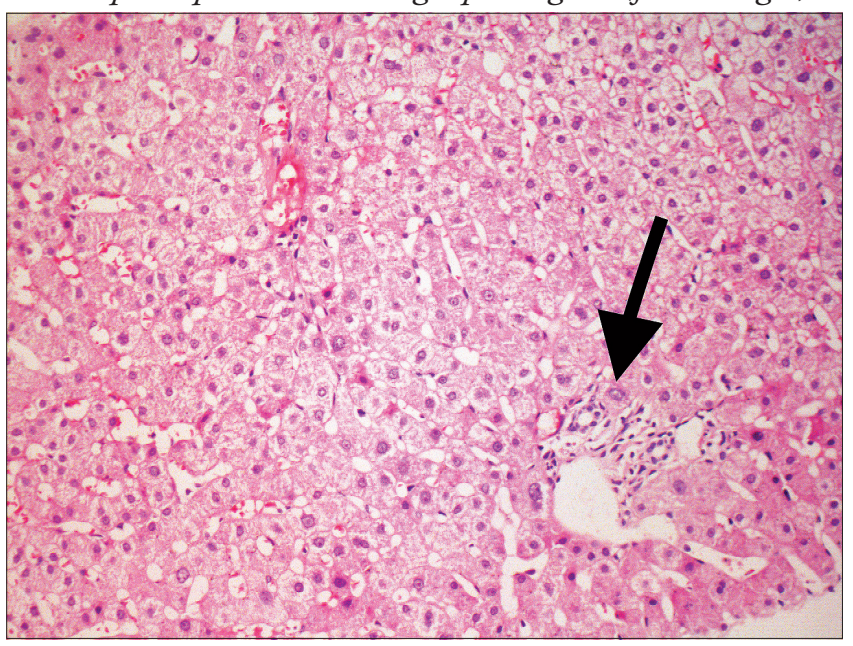

\section{Discusión}

La presentación clínica de la infección por el VHE es variable, abarca desde una forma subclínica hasta una hepatitis fulminante. ${ }^{1-6}$ En los pacientes inmunocomprometidos, puede evolucionar a cronicidad y en las mujeres embarazadas, existe un mayor riesgo de insuficiencia hepática cuando la infección es causada por el genotipo $10^{3,7-13}$ Clásicamente, se lo asoció a pacientes que viajaron a áreas endémicas como Asia o México, pero, actualmente, se sabe que el VHE genotipo 3 se encuentra en Argentina. ${ }^{14}$ Aunque nuestro país se considera un área de baja endemicidad, es posible que el VHE esté subdiagnosticado, por lo que debe descartarse en todos los pacientes que presenten una causa desconocida de he- patitis aguda. ${ }^{15,17}$ En los últimos años, en nuestro país, se ha detectado un aumento en la seroprevalencia de los autoanticuerpos IgG contra el VHE, siendo de un 16,7\% en los donantes de sangre y un 35\% en los pacientes con el virus de inmunodeficiencia humana $(\mathrm{VIH})$, según el último informe de Munné et al. en 2014. ${ }^{18}$ En esta publicación, también se informa sobre casos agudos de hepatitis secundaria al VHE asociados con el consumo de productos porcinos. ${ }^{18,19} \mathrm{El}$ aumento en la prevalencia del VHE en Argentina probablemente se asocie a la mejora en la sensibilidad de las pruebas de diagnóstico, así como a una mayor información disponible sobre esta etiología que hace que el diagnóstico sea más probable y favorezca la realización de las pruebas estandarizadas para el VHE en los pacientes con una hepatitis aguda. ${ }^{20,22}$

En publicaciones anteriores, se informó que los pacientes con hepatitis autoinmune tienen una mayor prevalencia del VHE. ${ }^{9,11,23}$ Esto ha llevado a la hipótesis de que la infección viral podría haber desencadenado y desarrollado una hepatitis autoinmune o que haya reacciones cruzadas en los pacientes con hepatitis autoinmune que causen una falsa reacción positiva de la serología del VHE. Por otro lado, también hay informes de casos de hepatitis aguda por el VHE que imitan la autoinmune. ${ }^{24,30} \mathrm{El}$ reporte mencionado previamente consta de dos casos, en los cuales el diagnóstico de hepatitis autoinmune se realizó mediante un puntaje positivo del IAHIG y en uno de estos pacientes, se inició un tratamiento inmunosupresor hasta que la serología del VHE fue positiva. Con este resultado, se detuvo el tratamiento con esteroides. ${ }^{24,25}$

En el caso que describimos, el diagnóstico inicial de hepatitis autoinmune fue respaldado por una puntuación del IAHIG simplificada de 7 puntos, basada en la presencia de autoanticuerpos séricos positivos, por niveles de IgG elevados y por hallazgos típicos en la biopsia hepática en ausencia de una infección por el virus de las hepatitis A, B o C..$^{31}$ Se inició el tratamiento con esteroides y azatioprina. Unas semanas después, recibimos el resultado positivo del VHE IgM, seguido de la detección de ARN del VHE positivo por la RT-PCR en muestras de sangre y heces, reduciendo así la puntuación simplificada del IAHIG a 5 puntos. Esto implicaba un diagnóstico menos probable de hepatitis autoinmune y favorecía el diagnóstico de hepatitis aguda por el VHE. Se decidió interrumpir el tratamiento inmunosupresor y se siguió al paciente con pruebas de laboratorio: enzimas hepáticas, anticuerpos autoinmunes contra la hepatitis, serología IgG e IgM VHE y ARN VHE por RT-PCR. En el seguimiento, las pruebas hepáticas se mantuvieron dentro del rango normal, los anticuerpos autoinmunes se volvieron negativos, hubo evidencia de seroconversión del VHE IgM a IgG 
y la carga viral del VHE se negativizó. También realizamos una biopsia hepática, un año después de suspender el tratamiento inmunosupresor, que no informó signos de hepatitis autoinmune ni de fibrosis (METAVIR F0). La diferencia entre la fibrosis de las biopsias podría deberse a que, en la hepatitis aguda, se observaba un edema colágeno, por lo que en ese momento se interpretó como F1, o bien deberse a un error de muestreo. Después de analizar todos estos datos y de acuerdo con los hallazgos clínicos, serológicos e histológicos, se estableció que nuestro paciente era un caso de hepatitis aguda por el virus E que imitaba una autoinmune.

\section{Conclusiones}

La hepatitis por el virus E está subdiagnosticada, en parte debido a la falta de sospecha en los pacientes con casos agudos. La hepatitis aguda por el virus $\mathrm{E}$ puede presentarse con síntomas clínicos, hallazgos de laboratorio e histológicos que se asemejan a una HAI, lo que conduce a un error de diagnóstico si no hay una búsqueda rutinaria de la serología VHE o RT-PCR. El diagnóstico de HAI comprende un tratamiento crónico con medicamentos que pueden tener reacciones adversas o efectos secundarios en el paciente, por lo que debe iniciarse solo si es realmente necesario. En general, en los pacientes con hepatitis aguda, el VHE debe descartarse antes de afirmar la HAI. Esto permitirá establecer un diagnóstico adecuado y evitará un tratamiento erróneo e innecesario con medicación inmunosupresora.

Agradecimientos. Dr. Fernando Gruz, médico hepatólogo. Dra. Gabriela Perli, médica anatomopatóloga.

Dr. Carlos Vigliano, médico anatomopatólogo.

Conflictos de intereses. Los autores declaran no tener conflictos de intereses.

Soporte financiero. Los autores no recibieron soporte $f i$ nanciero alguno para la realización de este manuscrito.

\section{Aviso de derechos de autor}

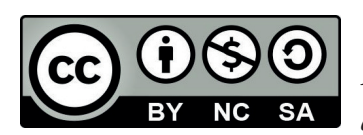

(C) 2021 Acta Gastroenterológica Latinoamericana. Este es un artículo de acceso abierto publicado bajo los términos de la Licencia Creative Commons Attribution (CC BY-NC-SA 4.0), la cual permite el uso, la distribución $y$ la reproducción de forma no comercial, siempre que se cite al autor y la fuente original.
Cite este artículo como: Garrido $M L$, Reyes Toso $M L$, Raffa $S$ y col. Hepatitis aguda por virus E con características de autoinmunidad. Reporte de un caso. Acta Gastroenterol Latinoam. 2021;51(2):217-22. https://doi.org/10.52787/ dtzy3014

\section{Referencias}

1. Mirazo S, Ramos N, Mainardi V, Gerona S, Arbiza J. Transmission, diagnosis, and management of hepatitis E: an update. Hepat Med. 2014;6:45-59.

2. Lanini S, Ustianowski A, Pisapia R, Zumla A, Ippolito G. Viral Hepatitis: Etiology, Epidemiology, Transmission, Diagnostics, Treatment, and Prevention. Infect Dis Clin North Am. 2019;33(4):1045-62.

3. Kamar N, Izopet J, Pavio N, Aggarwal R, Labrique A, Wedemeyer H, Dalton HR. Hepatitis E virus infection. Nat Rev Dis Primers. 2017;3:17086.

4. European Association for the Study of the Liver. EASL Clinical Practice Guidelines on hepatitis E virus infection. J Hepatol. 2018;68(6):1256-71.

5. Haffar S, Shalimar, Kaur RJ, Wang Z, Prokop LJ, Murad MH, Bazerbachi F. Acute liver failure caused by hepatitis E virus genotype 3 and 4: A systematic review and pooled analysis. Liver Int. 2018;38(11):1965-73.

6. Ramachandran J, Ramakrishna B, Eapen Eapen C, Abraham P, Zachariah UG, Jayram A, et al. Subacute hepatic failure due to hepatitis E. J Gastroenterol Hepatol. 2008;23(6):879-82.

7. Fainboim H, González J, Fassio E, Martínez A, Otegui L, Eposto $\mathrm{M}$, et al. Prevalence of hepatitis viruses in an anti-human immunodeficiency virus-positive population from Argentina. A multicentre study. J Viral Hepat. 1999;6(1):53-7.

8. Fantilli AC, Trinks J, Marciano S, Zárate F, Balderramo DC, Martínez Wassaf MG, et al. Unexpected high seroprevalence of hepatitis $\mathrm{E}$ virus in patients with alcohol-related cirrhosis. PLoS One. 2019;14(10):e0224404.

9. Eder M, Strassl R, Beinhardt S, Stättermayer AF, Kozbial K, Lagler H, et al. High seroprevalence of anti-Hepatitis E antibodies in Austrian patients with autoimmune hepatitis. Liver Int. 2019;39(4):640-5.

10. Van Gerven NMF, Van Der Eijk AA, Pas SD, Zaaijer HL, De Boer YS, Witte BI, et al. Seroprevalence of Hepatitis E Virus in Autoimmune Hepatitis Patients in the Netherlands. J Gastrointestin Liver Dis. 2016;25(1):9-13.

11. Le Cann P, Tong MJ, Werneke J, Coursaget P. Detection of antibodies to hepatitis $\mathrm{E}$ virus in patients with autoimmune chronic active hepatitis and primary biliary cirrhosis. Scand J Gastroenterol. 1997;32(4):387-9.

12. Wedemeyer H, Rybczynska J, Pischke S, Krawczynski K. Immunopathogenesis of hepatitis E virus infection. Semin Liver Dis. 2013;33(1):71-8.

13. Mateos-Lindemann ML, Diez-Aguilar M, Gonzalez Galdamez AL, Galán JC, Moreno A, Pérez-Gracia MT. Patients infected with HIV are at high-risk for Hepatitis E virus infection in Spain. J Med Virol. 2014;86(1):71-4.

14. Khuroo MS, Khuroo MS, Khuroo NS. Hepatitis E: Discovery, global impact, control and cure. World J Gastroenterol. 2016;22(31):7030-45. 
15. Munné MS, Vladimirsky S, Otegui L, Brajterman L, Castro R, Soto S, et al. Caracterización molecular del virus de hepatitis E en tres casos de falla hepática fulminante en niños de Argentina. Acta Gastroenterol Latinoam. 2006;36(3):125-30.

16. Echevarría JM, González JE, Lewis-Ximenez LL, Lopes Dos Santos DR, Munné MS, Pinto MA, Pujol FH, Rodríguez-Lay LA. Hepatitis E Virus Infection in Latin America: A Review. J Med Virol. 2013;85(6):1037-45.

17. Gruz F, Cleres M, Loredo D, Fernández MG, Raffa S, Yantorno S, et al. Hepatitis E: ¿Un virus infrecuente o un agente infrecuentemente pensado? Acta Gastroenterol Latinoam. 2013;43(2):143-5.

18. Munné MS, Altabert NR, Otegui MLO, Vladimirsky SN, Moreiro R, Espul MP, et al. Updating the knowledge of hepatitis E: New variants and higher prevalence of anti-HEV in Argentina. Ann Hepatol. 2014;13(5):496-502.

19. Munné MS, Vladimirsky S, Otegui L, Castro R, Brajterman L, Soto S, et al. Identification of the first strain of swine Hepatitis E virus in South America and prevalence of anti-HEV antibodies in swine in Argentina. J Med Virol. 2006;78(12):1579-83.

20. Arce LP, Müller MF, Martinez A, Baiker A, Marranzino G, Agote F, Vizoso-Pinto MG. A Novel In-House Enzyme-Linked Immunosorbent Assay for Genotype 3 Hepatitis E Virus Reveals High Seroprevalence in Blood Donors in Northern Argentina. Front Microbiol. 2019;10:2481.

21. Al-Absi ES, Al-Sadeq DW, Younis MH, Yassine HM, Abdalla OM, Mesleh AG, et al. Performance evaluation of five commercial assays in assessing seroprevalence of HEV antibodies among blood donors. J Med Microbiol. 2018;67(9):1302-9.

22. Avellon A, Morago L, Garcia-Galera MC, Munoz M, Echevarria JM. Comparative sensitivity of commercial tests for Hepatitis E genotype 3 virus antibody detection. J Med Virol. 2015;87(11):1934-9.
23. Pischke S, Gisa A, Suneetha PV, Wiegand SB, Taubert R, Schlue $\mathrm{J}$, et al. Increased HEV seroprevalence in patients with autoimmune hepatitis. PLoS One. 2014;9(1):e85330.

24. Nagasaki F, Ueno Y, Kanno N, Okamoto H, Shimosegawa T. A case of acute hepatitis with positive autoantibodies who actually had hepatitis E virus infection. Hepatol Res. 2005;32(2):134-7.

25. Nagasaki F, Ueno Y, Mano Y, Igarashi T, Yahagi K, Niitsuma $\mathrm{H}$, et al. A patient with clinical features of acute hepatitis $\mathrm{E}$ viral infection and autoimmune hepatitis. Tohoku J Exp Med. 2005;206(2):173-9.

26. Calisti G, Irish DN, Ijaz S, Tedder RS, Moore K. Acute hepatitis E mimicking a flare of disease in a patient with chronic autoimmune hepatitis. Annals of Hepatology. 2017;16:160-3.

27. Patel I, Companioni RC, Bansal R, Vyas N, Catalano C, Aron J, Walfish A. Acute hepatitis E presenting with clinical feature of autoimmune hepatitis. J Community Hosp Intern Med Perspect. 2016;6(6):33342.

28. Terziroli Beretta-Piccoli B, Ripellino P, Gobbi C, Cerny A, Baserga A, Di Bartolomeo C, et al. Autoimmune liver disease serology in acute hepatitis E virus infection. J Autoimmun. 2018;94:1-6.

29. Vieira CL, Baldaia C, Fatela N, Ramalho F, Cardoso C. Case of acute hepatitis $\mathrm{E}$ with concomitant signs of autoimmunity. World J Hepatol. 2013;5(3):152-5.

30. Minkoff NZ, Buzzi K, Williamson AK, Hagmann SHF. Case report: Acute hepatitis $\mathrm{E}$ in a pediatric traveler presenting with features of autoimmune hepatitis: A diagnostic and therapeutic challenge. Am J Trop Med Hyg. 2019;100(1):155-8.

31. European Association for the Study of the Liver. EASL Clinical Practice Guidelines: Autoimmune hepatitis. J Hepatol. 2015;63(4):971-1004. 